\title{
Advantages of Monte Carlo Confidence Intervals for Indirect Effects
}

\author{
Kristopher J. Preacher \\ Vanderbilt University \\ James P. Selig \\ University of New Mexico
}

\begin{abstract}
Monte Carlo simulation is a useful but underutilized method of constructing confidence intervals for indirect effects in mediation analysis. The Monte Carlo confidence interval method has several distinct advantages over rival methods. Its performance is comparable to other widely accepted methods of interval construction, it can be used when only summary data are available, it can be used in situations where rival methods (e.g., bootstrapping and distribution of the product methods) are difficult or impossible, and it is not as computer-intensive as some other methods. In this study we discuss Monte Carlo confidence intervals for indirect effects, report the results of a simulation study comparing their performance to that of competing methods, demonstrate the method in applied examples, and discuss several software options for implementation in applied settings.
\end{abstract}

In its simplest form, mediation occurs when the effect of an independent variable (X) on a dependent variable (Y) is transmitted via a mediator variable (M) (see Figure 1). ${ }^{1}$ This mediation effect is also commonly referred to as the indirect effect of $\mathrm{X}$ on $\mathrm{Y}$ through $\mathrm{M}$. Mediation models permit researchers to test simple hypotheses about how causal processes may occur and form the building blocks of more complicated structural models. Mediation models often involve parsing the total effect $(c)$ of $\mathrm{X}$ on $\mathrm{Y}$ into a direct effect $\left(c^{\prime}\right)$ and an indirect effect $(a \times b$, or simply $a b)$. These coefficients can be derived from fitting the following simultaneous equations to sample data using linear regression or path analysis, with straightforward extensions to latent variable models:

$$
\begin{gathered}
\mathrm{M}=a_{0}+a \mathrm{X}+\mathrm{e}_{\mathrm{M}} \\
\mathrm{Y}=b_{0}+b \mathrm{M}+c^{\prime} \mathrm{X}+\mathrm{e}_{\mathrm{Y}}
\end{gathered}
$$

Figure 1 depicts these effects using simple path diagrams of the effect of $\mathrm{X}$ on $\mathrm{Y}$ both without and with $\mathrm{M}$ included in the model. In mediation analysis, attention focuses mostly on the indirect

\footnotetext{
${ }^{1}$ In order for the results of a mediation analysis to have a causal interpretation, several additional assumptions must be met. For overviews see Imai, Keele, and Tingley (2010), Muthén (2011), and Pearl (2010).

Correspondence should be addressed to Kristopher J. Preacher, Department of Psychology \& Human Development, Vanderbilt University, PMB 552, 230 Appleton Place, Nashville, TN 37203-5721. E-mail: kris.preacher@ vanderbilt.edu
} 


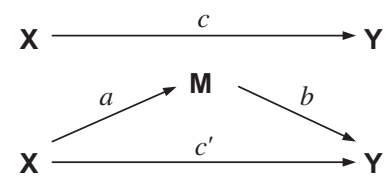

FIGURE 1 A simple mediation model. $\mathrm{X}$ is the predictor, $\mathrm{M}$ the hypothesized mediator, and $\mathrm{Y}$ the outcome. Regression residuals are excluded from the diagram for simplicity.

effect $a b$, although many methodologists emphasize the need to also consider the coefficients $c$ and $c^{\prime}$ in making this determination. In more complicated mediation models, such as models with multiple mediators, hierarchically clustered data, or longitudinal data, the indirect effect expression will be correspondingly more complex.

In this article we discuss Monte Carlo (MC) confidence intervals for indirect effects. MC intervals capitalize on the fact that most statistics software packages provide the asymptotic covariance matrix of the parameter estimates involved in indirect effects. A sampling distribution of the indirect effect can be simulated from this matrix, and percentiles of this distribution can be used to construct a confidence interval. We provide a small simulation study comparing the performance of MC confidence intervals to parametric, nonparametric, and residual-based bootstrap intervals, as well as intervals based on the delta method and distribution of products, demonstrate the method in two examples, and discuss several software options for implementation in applied settings.

\section{METHODS FOR CONSTRUCTING CONFIDENCE INTERVALS FOR INDIRECT EFFECTS}

Let $\theta$ be a generic population parameter whose value we wish to estimate. Chernick (2008) defines a $100(1-\alpha) \%$ confidence interval (CI) for the value of $\theta$ in the following way:

“. . . if we construct a $95 \%$ confidence interval, we would expect that our procedure would produce intervals that contain the true parameter in $95 \%$ of the cases. Such is the definition of a confidence interval." (p. 53)

Thus, a CI for $\theta$ may be defined in terms of the true (unknown) parameter value without reference to any particular null hypothesis. A simple example of a confidence interval is a $95 \%$ CI for the population mean of a normally distributed variable, expressed as $\mathrm{CI}_{.95}:\left\{\hat{\theta} \pm t_{c} S E_{\hat{\theta}}\right\}$, where $t_{c}$ is the two-tailed critical value of the corresponding Student's $t$ distribution and $S E_{\hat{\theta}}$ is the standard error of the estimate. With reference to the model in Figure 1, the indirect effect is most often quantified as the sample estimate $\hat{a} \hat{b}$. Various methods for constructing CIs for the parameter $a b$ differ only in how the sampling distribution of $\hat{a} \hat{b}$ is obtained and used to gauge significance. We next describe the major methods of CI construction for indirect effects.

\section{Delta Method}

The delta method (DM) is a popular method for deriving the sampling variance of compound statistics like $\hat{a} \hat{b}$. This sampling variance, in turn, can be used in the construction of symmetric 
confidence intervals. The method proceeds by first obtaining the asymptotic covariance matrix of parameter estimates $\hat{a}$ and $\hat{b}$, then pre- and post-multiplying by the vector-valued derivative (D) of the Taylor series expansion of $\hat{a} \hat{b}$ with respect to each of these parameters evaluated at their means. The result is a scalar approximation to the sampling variance of $\hat{a} \hat{b}$ where $s_{\hat{b}}^{2}$ and $s_{\hat{a}}^{2}$ are the estimated sampling variances of $\hat{a}$ and $\hat{b}$, respectively:

$$
\operatorname{var}[\hat{a} \hat{b}] \approx \hat{a}^{2} s_{\hat{b}}^{2}+\hat{b}^{2} s_{\hat{a}}^{2}
$$

The square root of this sampling variance is often used as a standard error in significance tests for the indirect effect (Sobel, 1982) or to construct a symmetric confidence interval for $a b$ :

$$
\mathrm{CI}_{1-\alpha}=\hat{a} \hat{b} \pm z_{\alpha / 2} \sqrt{\operatorname{var}[\hat{a} \hat{b}]}
$$

Unfortunately, $\hat{a} \hat{b}$ is not normally distributed, although it may approach normality in large samples (Aroian, 1947; Craig, 1936). In most cases, the distribution of $\hat{a} \hat{b}$ is skewed and leptokurtic (Bollen \& Stine, 1990; MacKinnon, Lockwood, Hoffman, West, \& Sheets, 2002; MacKinnon, Lockwood, \& Williams, 2004). When the assumption of normality is not tenable, intervals based on the delta method are not appropriate in the majority of cases. Nevertheless, the delta method is the default method most commonly included in structural equation modeling (SEM) software for obtaining tests and CIs for indirect effects.

\section{Distribution of the Product Method}

The distribution of the product (DP) strategy considers the correct distribution of $\hat{a} \hat{b}$ rather than assuming that it is normal or approximating it in some way. To use the DP method, researchers are obliged to convert $\hat{a}$ and $\hat{b}$ to $z$-scores, form the product $z_{\hat{a}} z_{\hat{b}}$, and compare the result to a table of critical values (MacKinnon et al., 2004; Meeker, Cornwell, \& Aroian, 1981). This method performs well in simulation studies, but until recently required recourse to tables with limited availability and knowledge of the population value of either $a$ or $b$. The first of these limitations has been overcome with the availability of PRODCLIN (available in Fortran, SAS, SPSS, and R formats; MacKinnon, Fritz, Williams, \& Lockwood, 2007), a program that can be used to construct asymmetric confidence limits using the distribution of the product approach. The second limitation cannot be circumvented but becomes less relevant as $N$ increases.

\section{Nonparametric Percentile Bootstrap and Corrections}

Bootstrapping (Efron, 1982; Efron \& Tibshirani, 1993) is a nonparametric resampling method that involves generating an empirical sampling distribution of $a b$ and using it as a basis for statistical estimation and inference. An arbitrarily large number $(B)$ of resamples of size $N$ are sampled independently with replacement from the original sample. The product $a b^{*}$ is estimated for each of these $B$ resamples, resulting in an empirical sampling distribution of $a b^{*}$. Percentile-based (PC) confidence intervals (Efron, 1981) may be constructed for any degree of precision by identifying the values of $a b^{*}$ corresponding to the lower and upper $50 \alpha \%$ of the distribution. These values define the limits of a $100(1-\alpha) \%$ asymmetric CI about the sample $\hat{a} \hat{b}$. 
Efron (1981, 1982, 1987) and Efron and Tibshirani (1986, 1993) suggest adjustments that reduce bias in percentile bootstrap interval bounds. Whereas percentile bounds are taken directly from the desired percentiles of the empirical sampling distribution of $a b^{*}$, bias-corrected (BC) bounds incorporate an adjustment. Letting $z_{0}$ be the $z$-score corresponding to the proportion of the $B$ bootstrap resamples with $a b^{*}$ less than the estimated sample $\hat{a} \hat{b}$, two $z$-scores are defined as:

$$
\begin{gathered}
z_{\text {lower }}^{\prime}=2 z_{0}+z_{\alpha / 2} \\
z_{\text {upper }}^{\prime}=2 z_{0}+z_{1-\alpha / 2}
\end{gathered}
$$

The proportions under the standard normal distribution that correspond to $z_{\text {lower }}^{\prime}$ and $z_{\text {upper }}^{\prime}$ are multiplied by 100 to serve as the adjusted percentiles for selecting interval limits from the bootstrap distribution of $a b^{*}$. The BC bootstrap has been found to perform well in terms of Type I error rates and statistical power when testing indirect effects (MacKinnon et al., 2004). However, it has been observed that BC limits do not always have good coverage (Schenker, 1985); that is, across repeated sampling, $\mathrm{BC}$ intervals do not necessarily include the population parameter the desired percentage of times. This in part motivated the creation of bias-corrected and accelerated (BCa) confidence limits (Efron, 1987). BCa percentiles include further adjustment by an acceleration constant $\dot{a}$ :

$$
\begin{gathered}
z_{\text {lower }}^{\prime}=z_{0}+\frac{z_{0}+z_{\alpha / 2}}{1-\dot{a}\left(z_{0}+z_{\alpha / 2}\right)} \\
z_{\text {upper }}^{\prime}=z_{0}+\frac{z_{0}+z_{1-\alpha / 2}}{1-\dot{a}\left(z_{0}+z_{1-\alpha / 2}\right)}
\end{gathered}
$$

where $\dot{a}$ is approximately $1 / 6$ of the skewness of the bootstrap distribution of $a b^{*}$. Thus, $2 z_{0}$ is a correction for median bias and $\dot{a}$ is a correction for skewness (DiCiccio \& Efron, 1996; Efron \& Tibshirani, 1986, 1993). BCa confidence limits are second-order accurate in many cases where estimators can be expressed as functions of multivariate vector means (e.g., indirect effects) (DiCiccio \& Romano, 1988; Efron, 1987, p. 176; Efron \& Tibshirani, 1993; Hall, 1989), meaning that coverage rates approach the nominal value very quickly as $N$ increases. In theory, $\mathrm{BCa}$ intervals offer an improvement over BC intervals, but in practice the superiority of one type of interval over the other can depend on the context.

The primary benefits of bootstrapping are that it involves no distributional assumptions and can be used in small samples. Unlike DM intervals, bootstrap intervals for $a b$ are asymmetric, resembling more closely the true distribution of products, which tends to be somewhat skewed away from zero (Bollen \& Stine, 1990). The only apparent drawbacks to the bootstrap include slight inconsistency among replications of the same experiment with the same data due to random resampling variability and the time commitment due to computer-intensive resampling.

Bootstrapping has been found to perform well relative to other methods of CI construction for indirect effects (Bollen \& Stine, 1990; Efron \& Tibshirani, 1993; Lockwood \& MacKinnon, 1998; 
MacKinnon et al., 2004; Preacher \& Hayes, 2004; Shrout \& Bolger, 2002). Hypothesis tests based on BC and BCa intervals have fairly accurate Type I error rates and higher power when compared to competing methods used to test null hypotheses about indirect effects. Fritz and MacKinnon (2007) demonstrated that, of several competing methods, BC required the smallest $N$ to achieve comparable levels of power. On the other hand, Fritz and MacKinnon (2007), Fritz, Taylor, and MacKinnon (2012), and MacKinnon et al. (2004) report that BC and BCa bootstrap CIs yield too-high Type I error rates in some conditions when $N$ is small, and Polansky (1999) reports poor CI coverage in very small samples. Biesanz, Falk, and Savalei (2010) found that BCa intervals did not perform as well as PC intervals. Bootstrap methods are available for estimating indirect effects in some SEM software applications and in macros for use in SPSS and SAS (Cheung, 2007; Lockwood \& MacKinnon, 1998; Preacher \& Hayes, 2004, 2008; Preacher, Rucker, \& Hayes, 2007; Shrout \& Bolger, 2002).

\section{Residual-Based Bootstrap}

Residual-based bootstrapping (RB) is often used with regression models in which the design matrix is considered fixed, which includes most applications of regression (Efron, 1979; Efron \& Tibshirani, 1993; Freedman, 1981; Stine, 1989). It has been adapted for use in multilevel modeling where there is no clearly superior way to employ more traditional bootstrap methods (van der Leeden, Meijer, \& Busing, 2008; Wang, Carpenter, \& Kepler, 2006). In the regression or SEM context, the first step in residual-based bootstrapping is to estimate the regression weights or path coefficients and compute observed residuals $\left(\hat{e}_{\mathrm{M}}\right)$ according to, for example, Equation 1. The second step is to bootstrap the residuals. The resampled residuals are then added to the fitted equation $\hat{a}_{0}+\hat{a} \mathrm{X}$ to generate bootstrap values of the mediator $\mathrm{M}^{*}$. These, in turn, are regressed onto the fixed $\mathrm{X}$ to produce a bootstrapped value of $a^{*}$. This process is repeated $B$ times to generate a bootstrapped distribution of $a^{*}$, where $B$ is large (several thousand). In adapting the method to the simple mediation context, there are two residuals to resample: those for the $\mathrm{M}$ equation and those for the Y equation (Zhang \& Wang, 2008). For simple mediation models, pairs of residuals $\left(\hat{e}_{\mathrm{M}}, \hat{e}_{\mathrm{Y}}\right)$ are obtained from Equations 1 and 2 fit to the observed data. Residual pairs are then bootstrapped from this joint distribution and added (respectively) to the fitted equations $\hat{a}_{0}+\hat{a} \mathrm{X}$ and $\hat{b}_{0}+\hat{b} \mathbf{M}+\hat{c}^{\prime} \mathrm{X}$ to yield bootstrapped values of both $\mathbf{M}^{*}$ and $\mathrm{Y}^{*} . \mathrm{M}^{*}$ is regressed on the fixed $\mathrm{X}$ to yield $a^{*}$, whereas $\mathrm{Y}^{*}$ is regressed on the fixed $\mathrm{X}$ and the random $\mathrm{M}^{*}$ to yield $b^{*}$. This process is repeated $B$ times to generate a bootstrap distribution of $a b^{*}$. The empirical sampling distribution of $a b^{*}$ can be used in the usual way to form PC, BC, or BCa CIs. Zhang and Wang (2008) note that RB bootstrap requires errors to be independent and identically distributed (iid), an assumption not required for other bootstrapping methods. They found that RB bootstrap CIs have good coverage and power for medium to large effects when residuals are iid. When residuals are heterogeneous, the RB bootstrap performed better with small effect size whereas the PC bootstrap performed better with large effect sizes.

The RB bootstrap has been used to assess mediation in multilevel models (Pituch, Stapleton, $\&$ Kang, 2006) but has only recently been suggested for use in the more common single-level mediation models (Zhang \& Wang, 2008). The version of the RB bootstrap used in our simulation is identical to that of Zhang and Wang and similar to that of Pituch et al., except that residuals were obtained via bootstrapping rather than through Monte Carlo simulation. Zhang and Wang provide software (MedCI) for computing confidence limits using the RB bootstrap. 


\section{Parametric Bootstrap}

The parametric bootstrap (PB) method can be traced to Efron (1980, 1982; Efron \& Tibshirani, 1986, 1993), and is sometimes called parametric simulation (Davison \& Hinkley, 1997). The PB method relies on the assumption that the parameters $a$ and $b$ have a known joint sampling distribution, not necessarily normal, with parameters supplied by (often, but not necessarily) maximum likelihood estimates from the fitted parametric model. The model, with parameter estimates treated as parameters, is used to repeatedly generate bootstrap resamples. For example, the model-implied covariance matrix in a linear path model might be used as a population matrix, from which sample data matrices could be generated (e.g., using the method of Kaiser and Dickman [1962]). The statistic of interest $(\hat{\theta})$, which may be a simple or complex function of other model statistics, is computed in each resample. Thus, in contrast to the nonparametric bootstrap, in which bootstrap data consist of resampled cases from the original sample, in the parametric bootstrap the bootstrap data are generated parametrically from a fitted model. Percentiles of the sampling distribution of $\hat{\theta}$ are identified to serve as limits for a $100(1-\alpha) \%$ asymmetric confidence interval about the sample $\hat{\theta}$, where $\hat{\theta}=\hat{a} \hat{b}$ in mediation analysis. Bias correction, with or without acceleration, may be used just as with the nonparametric bootstrap.

Generation of the parametrically simulated data can proceed in a number of ways, typically by employing software-based pseudorandom number generation. Even though parametric assumptions are invoked for $\hat{a}$ and $\hat{b}$, no parametric assumptions are made about the distribution of $\hat{a} \hat{b}$. The advantages of the PB method include most of those associated with nonparametric bootstrap CIs - the intervals are properly asymmetric, and are useful in situations where obtaining CIs by analytic means is difficult or impossible. An advantage over the nonparametric bootstrap is that the PB method is not limited to using case data that were actually observed in one's empirically obtained sample; data are generated parametrically, which implies that the sampling distribution of $\hat{a} \hat{b}$ will be smoother. This becomes important especially when high confidence levels are desired (e.g., 99\%), the sample is small (Davison \& Hinkley, 1997), and/or the sampling distribution is heavily skewed. Furthermore, the PB method can be used when only summary data are available, facilitating re-analysis or meta-analysis. A disadvantage is that it is necessary to make some possibly unwarranted parametric distributional assumptions at the data simulation stage. The procedure will yield biased results to the extent that the original point estimates and their asymptotic (co)variances are affected by model misspecification.

Accessible discussions of the PB method can be found in Chernick (2008, pp. 124-125), Davison and Hinkley (1997, pp. 15-21), Efron (1980, pp. 40-41; 1982, pp. 29-30; 1986, pp. 56-57), and Efron and Tibshirani (1993, pp. 53-56). The PB method is implemented in the R command "boot" using sim="parametric" (Davison \& Hinkley, pp. 528-529).

\section{The Monte Carlo Method}

The Monte Carlo (MC) method involves generating a sampling distribution of a compound statistic by using point estimates of its component statistics, along with the asymptotic covariance matrix of these estimates and assumptions about how the component statistics are distributed. 
For example, a sampling distribution for the ratio of two independent means could be generated by first fitting a model to empirical data and obtaining point estimates and asymptotic variances for the means. Because means are asymptotically normal according to the Central Limit Theorem, a large number of random draws could be taken from a bivariate normal distribution of the means, each time creating the ratio $\theta^{*}=\bar{x}_{1}^{*} / \bar{x}_{2}^{*}$, yielding a sampling distribution of the ratio. A CI can then be formed on the basis of this sampling distribution in the same way as bootstrap intervals. However, in contrast to bootstrap methods, the MC method involves directly generating sample statistics from their joint asymptotic distribution, not resampling or generating data.

The MC method was first applied to the mediation context by MacKinnon et al. (2004) and is closely related to the empirical-M method (MacKinnon et al., 2002, 2004; Pituch \& Stapleton, 2008; Pituch et al., 2006; Pituch, Whittaker, \& Stapleton, 2005), especially as generalized by Williams and MacKinnon (2008) to cases beyond single-mediator models. The MC method relies on the assumption that the parameters $a$ and $b$ have a joint normal sampling distribution, with parameters supplied by (often, but not necessarily) maximum likelihood estimates from the fitted parametric model:

$$
\left[\begin{array}{l}
a^{*} \\
b^{*}
\end{array}\right] \sim \operatorname{MVN}\left(\left[\begin{array}{l}
\hat{a} \\
\hat{b}
\end{array}\right],\left[\begin{array}{cc}
\hat{\sigma}_{\hat{a}}^{2} & \hat{\sigma}_{\hat{a} \hat{b}} \\
\hat{\sigma}_{\hat{a} \hat{b}} & \hat{\sigma}_{\hat{b}}^{2}
\end{array}\right]\right)
$$

In the traditional three-variable mediation model of Figure $1, \hat{\sigma}_{\hat{a} \hat{b}}$ is often replaced with 0 for simplicity. Using the parametric assumption in (9), a sampling distribution of $\hat{a} \hat{b}$ is formed by repeatedly generating $a^{*}$ and $b^{*}$ and computing their product. Values for $\hat{a}$ and $\hat{b}$ can be generated in a number of ways, most often using software-based pseudorandom number generation. Parametric assumptions are invoked for $\hat{a}$ and $\hat{b}$, but no parametric assumptions are made about the distribution of $\hat{a} \hat{b}$. Even though parametric assumptions are invoked for $\hat{a}$ and $\hat{b}$, no parametric assumptions are made about the distribution of $\hat{a} \hat{b}$. Percentiles of this sampling distribution are identified to serve as limits for a $100(1-\alpha) \%$ asymmetric confidence interval about the sample $\hat{a} \hat{b}$.

Advantages of the MC method include most of those associated with nonparametric bootstrap CIs (e.g., asymmetry, usefulness in otherwise intractable situations). It also shares the advantage of the PB method in that the sampling distribution of $\hat{a} \hat{b}$ can be made arbitrarily smooth as $B$ increases, and it can be used when only summary data are available. The MC method has a unique advantage over bootstrap methods in that it is very fast; the model is fit to data only once. This can be a huge advantage when using models that take a long time to converge. The MC method can be used in situations where bootstrapping is not feasible, such as multilevel modeling, when samples are so small that bootstrapping may randomly generate a constant variable and stop, or when distinct values of a variable are limited and highly unbalanced (as in research on rare disorders). MacKinnon et al. (2004) found MC CIs to show performance similar to the PC bootstrap and inferior to BC bootstrap CIs. Thus, if the MC method is found to perform similarly to (or not significantly worse than) bootstrap methods and the DP method, we argue that researchers should give it serious consideration as a viable alternative to these methods. 


\section{SIMULATION STUDY}

To investigate the performance of Monte Carlo CIs relative to bootstrap-based CIs, delta method CIs, and CIs based on the distribution of products, we conducted a simulation study. A CI construction method is deemed successful to the extent that it demonstrates (a) nominal coverage, (b) smaller width, and (c) roughly equal numbers of "misses" to the left and to the right. That is, a good CI should capture the parameter $100(1-\alpha) \%$ of the time, should be narrower than CIs constructed using competing methods (holding coverage constant), and ideally should yield intervals that exclude the population parameter $50 \alpha \%$ to the left and $50 \alpha \%$ to the right (MacKinnon et al., 2004).

With these goals in mind, we chose a simple two-path mediation model (like the model depicted in Figure 1) for the simulation, with the expectation that results for this model will also apply to more complex models. We manipulated sample size $(N=20,40,70,100,150$, 200), effect sizes of the path coefficients in a simple three-variable mediation model $(a=.00$, $.14, .39, .59 ; b=.00, .14, .39, .59 ; c=.35, .70$ ), and CI construction method (DM, DP, PC, BCa, $\mathrm{BC}, \mathrm{RB}, \mathrm{PB}, \mathrm{MC})$. Population variances of $\mathrm{X}, \mathrm{M}$, and $\mathrm{Y}$ were chosen as 1.0, and the values of $a$ and $b$ were selected to conform to values used in prior simulation studies (e.g., Biesanz et al., 2010; MacKinnon et al., 2004). Two thousand samples were generated for each crossing of $N, a$, $b$, and $c$, yielding a total of 192,000 data sets. Nonparametric bootstrap methods used $B=1,000$ bootstrap resamples for each sample, the parametric bootstrap used 1,000 simulated data sets, and the Monte Carlo method used 1,000 simulated parameter sets.

\section{Hypotheses}

\section{Coverage}

Given the known inability of the delta method to accommodate asymmetry in the sampling distribution of an indirect effect, it was hypothesized that the delta method would perform poorly with respect to coverage. We expected all other methods to perform generally better than the delta method and acceptably overall, especially as sample size increased. We expected the Monte Carlo method to perform about as well as the nonparametric bootstrap methods and the distribution of the product method.

\section{Cl width}

Assuming that a CI method yields CIs with acceptable coverage, narrow confidence intervals are generally preferred to wider ones. The delta method is expected to yield relatively narrow intervals because it involves assuming normality for a sampling distribution that is actually skewed and leptokurtic (Bollen \& Stine, 1990; MacKinnon et al., 2002; MacKinnon et al., 2004). The expected narrowness of CIs based on the delta method is not a sign of superiority, but rather a sign of a parametrically misspecified sampling distribution. CIs based on bootstrap methods, Monte Carlo, and the distribution of the product are expected to yield CIs with comparable width. 


\section{Misses}

Ideally, a CI should exclude the parameter to an equal degree to the left and right; that is, it should be symmetric with respect to misses. To operationalize this symmetry, we computed the ratio of right-side misses to left-side misses. A value of 1 indicates perfect symmetry with respect to missing the population indirect effect, whereas departures from 1 indicate imbalance. We expected the delta method to yield unbalanced misses due to its artificially symmetric CIs. We expected Monte Carlo methods to perform on par with bootstrap and distribution of the product methods.

Overall, it was our expectation that the Monte Carlo method would perform acceptably on all counts when compared to bootstrap methods and the distribution of the product method. If the Monte Carlo method is comparable to (or not much worse than) existing methods for constructing asymmetric CIs, we argue that this method presents a useful alternative to these other methods when raw data are unavailable.

\section{Results}

\section{Coverage}

Coverage is reported for all cells of the design in Figure 2 (for $c=.35$ ) and Figure 3 (for $c=.70$ ). Overall, results were comparable for $c=.35$ and $c=.70$, and results for the $\mathrm{BC}$ and $\mathrm{BCa}$ bootstrap methods were virtually indistinguishable. As predicted, the delta method tended to have coverage that was too high in some conditions, mainly when either $a$ or $b$ was small. As $a$ and $b$ increased, and as sample size increased, most methods converged on .95 . The Monte Carlo method appeared to perform comparably to the parametric, percentile, and residual-based bootstrap methods. To better quantify coverage, we computed the average root mean squared error (RMSE) of observed CI coverage (with respect to the nominal .95 level) across all $a, b$, and $N$ conditions (but separately for $c=.35$ and $c=.70$ ). Results, reported in Table 1, demonstrate that the Monte Carlo method was among the best methods in terms of coverage-as good as, or slightly better than - the parametric, percentile, and residual-based bootstrap methods and the distribution of the product method.

\section{Cl width}

Summary results for interval width are reported in Table 1. Averaging across all conditions, the delta method showed the narrowest intervals (average width $=.233$ ), although this narrowness is of questionable worth given its relatively poor coverage. Among the remaining methods with acceptable coverage, the distribution of the product method had the narrowest CIs (.236), followed by the Monte Carlo method (.249).

\section{Misses}

The ratio of average right-side misses to average left-side misses is reported for all methods in Table 1. All methods demonstrated more average misses to the right than to the left (keeping in 

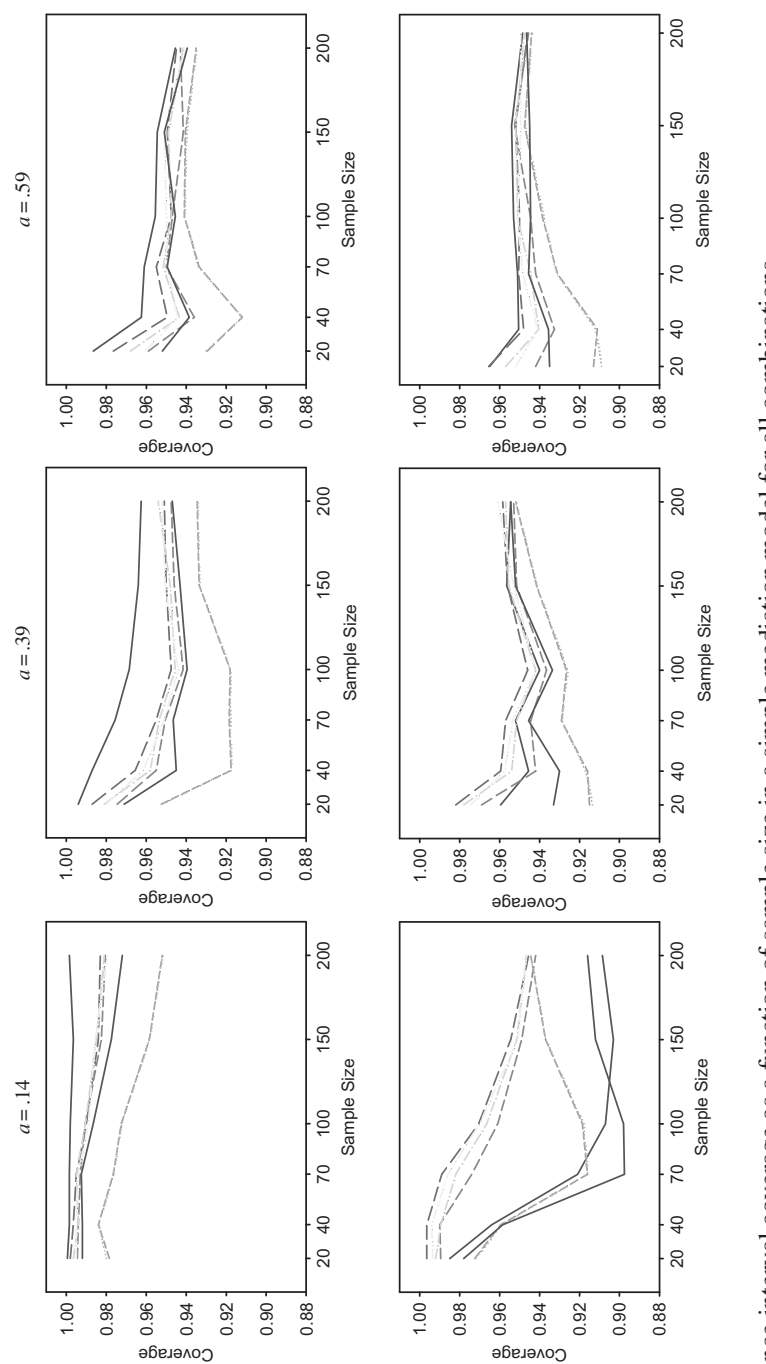

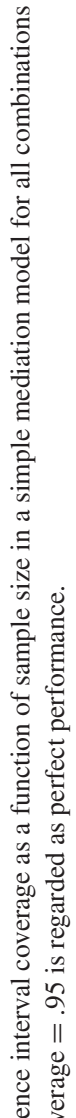
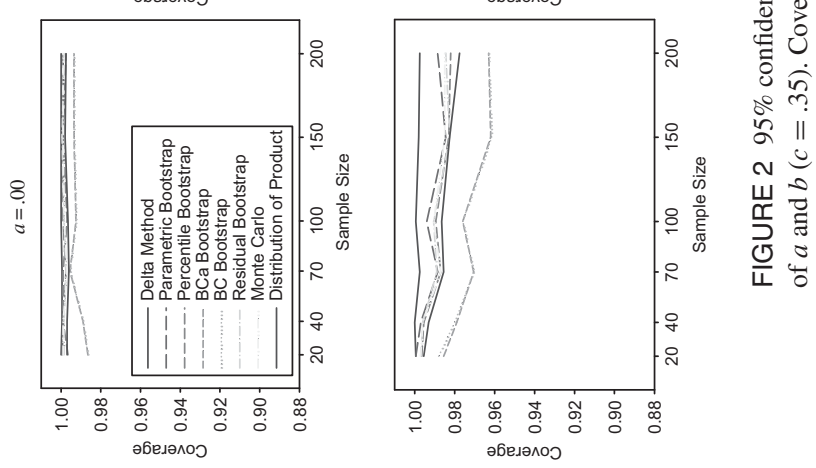

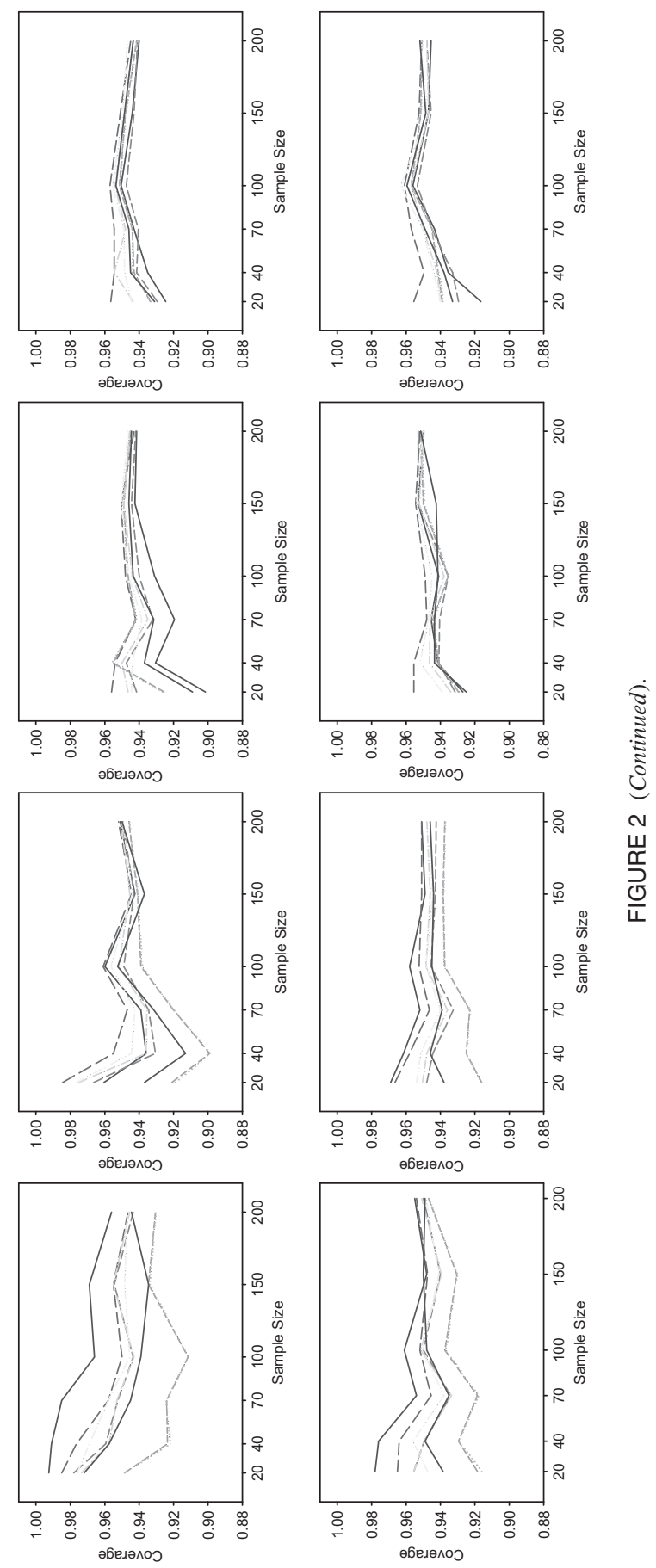

$6 \varepsilon^{*}=q$

$6 S^{\circ}=q$ 

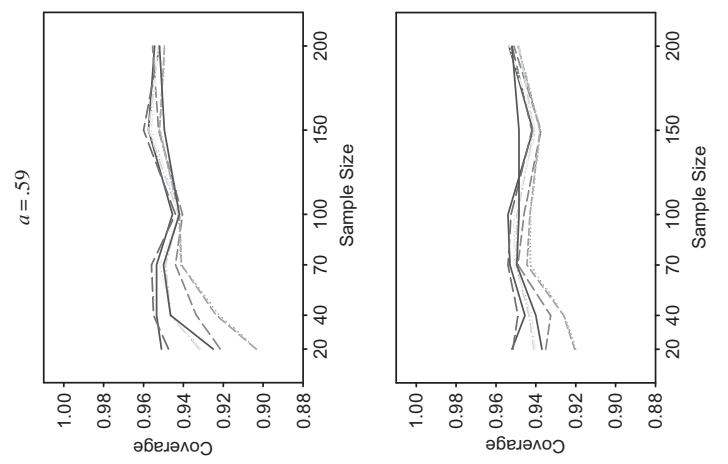

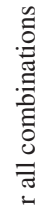
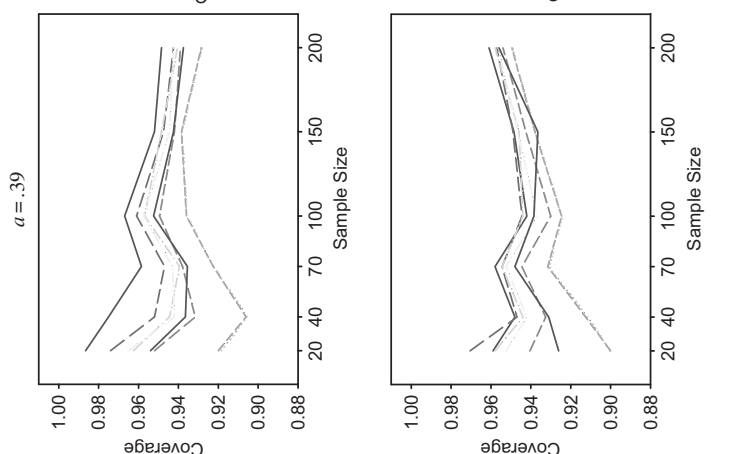

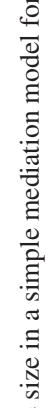
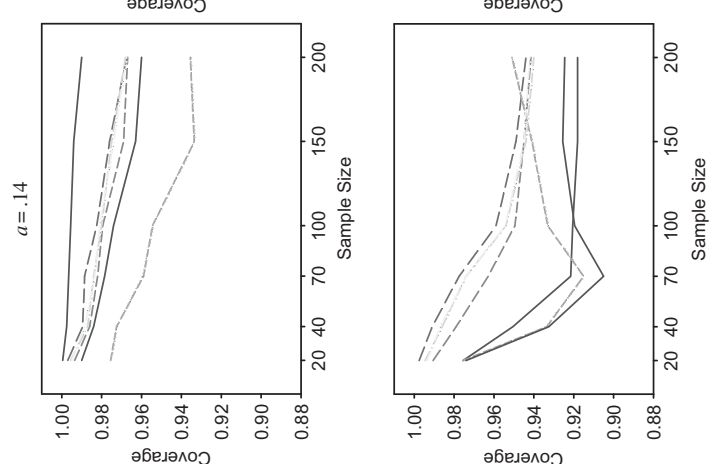

츨

颉

ำ

氙

वै

:

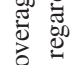

용

ำ

.

ญ

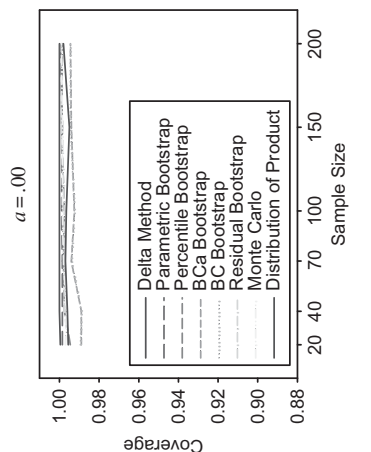

$00^{\circ}=q$

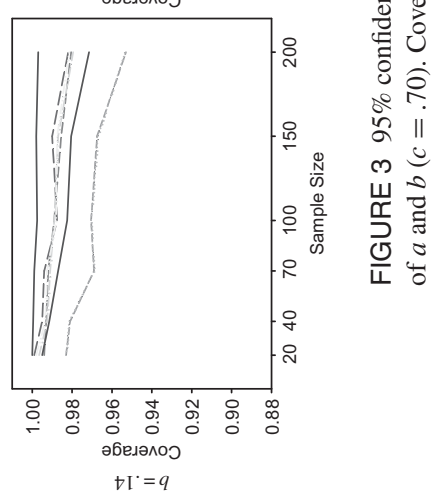



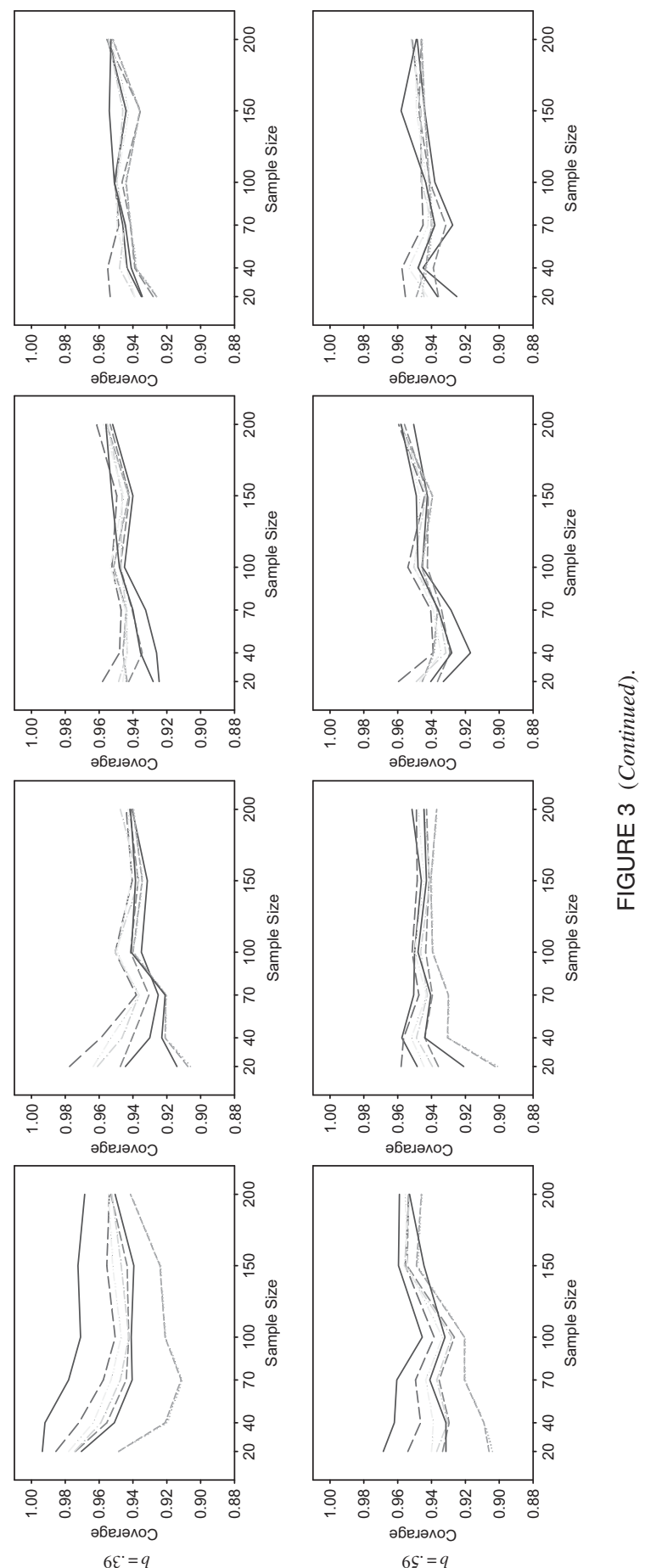
TABLE 1

Abbreviated Simulation Results

\begin{tabular}{lcccccc}
\hline & $\begin{array}{c}\text { RMSE for } \\
\text { Coverage } \\
(c=.35)\end{array}$ & $\begin{array}{c}\text { RMSEfor } \\
\text { Coverage } \\
(c=.70)\end{array}$ & $\begin{array}{c}\text { Overall } \\
\text { Width } \\
\text { for CIs }\end{array}$ & $\begin{array}{c}\text { Proportion } \\
\text { of Misses } \\
\text { to Left }\end{array}$ & $\begin{array}{c}\text { Proportion } \\
\text { of Misses } \\
\text { to Right }\end{array}$ & $\begin{array}{c}\text { Right- } \\
\text { to-Left } \\
\text { Miss Ratio }\end{array}$ \\
\hline Delta Method & .028 & .025 & .233 & .011 & .030 & 2.743 \\
Parametric Bootstrap & .023 & .021 & .259 & .015 & .022 & 1.449 \\
Percentile Bootstrap & .021 & .021 & .252 & .019 & .027 & 1.467 \\
BCa Bootstrap & .023 & .023 & .257 & .025 & .032 & 1.271 \\
BC Bootstrap & .023 & .023 & .257 & .025 & .032 & 1.272 \\
Residual Bootstrap & .022 & .020 & .252 & .017 & .025 & 1.450 \\
Monte Carlo & .022 & .020 & .249 & .017 & .025 & 1.447 \\
Distribution of Product & .023 & .021 & .236 & .019 & .031 & 1.670 \\
\hline
\end{tabular}

mind that the population indirect effect was positive when present). The delta method was by far the most unbalanced in terms of misses, with a right-to-left ratio of 2.743; that is, the population $a b$ was nearly three times more likely to be excluded to the right of the interval than to the left. The Monte Carlo method performed comparably to the bootstrap methods and better than the distribution of the product method.

Overall, the Monte Carlo method received much support. Its performance in terms of coverage, CI width, and misses was comparable to other top-performing methods of CI construction.

\section{EMPIRICAL EXAMPLES}

Next we provide two examples based on the results from an experiment conducted by Tal-Or, Cohen, Tsfati, and Gunther (2010). We will illustrate the utility of the MC approach by estimating CIs for both a simple indirect effect and a more complex total indirect effect using only the information available in the published report. Tal-Or and colleagues allowed us to use the original data from their experiment so it will be possible to make comparisons between MC CIs based only on summary statistics and delta method and bootstrap CIs based on the analysis of the original data.

We will focus on results from Study 2 in Tal-Or et al. (2010). In this study, the authors examined the relationship between an experimental manipulation of the perceived impact of a simulated newspaper story $(\mathrm{X})$ on the participants' potential reactions to the story $(\mathrm{Y})$. Two variables, the presumed influence of the story on the public $\left(\mathrm{M}_{1}\right)$, and the perceived importance of the story $\left(\mathrm{M}_{2}\right)$, were examined as mediators. The perceived impact of the story was operationalized by placing the story on the front page or on an internal page of a supplement to the newspaper. The other variables were measured with questionnaires. Figure 4 shows a diagram of the four variables in the study. We added subscripts to the respective $a$ and $b$ paths to better distinguish the two sets of coefficients. Tal-Or et al. assessed mediation using a combination of Baron and Kenny's (1986) causal steps approach and bootstrap confidence intervals. The CIs were not reported, but the authors used the CIs to determine that both indirect effects depicted in Figure 4 
(i.e., perceived impact $\rightarrow$ media influence $\rightarrow$ potential reactions and perceived impact $\rightarrow$ perceived importance $\rightarrow$ potential reactions) were statistically significant $(p<.05)$.

For the example of simple mediation, we will examine the effect of perceived impact on potential reactions as mediated by presumed media influence. Tal-Or and colleagues report both the coefficients and standard errors for this indirect effect $\left(\hat{a}_{1}=0.48, S E_{a 1}=0.24 ; \hat{b}_{1}=0.40\right.$, $\left.S E_{b 1}=0.09\right)$. We entered these summary statistics into a web-based Monte Carlo calculator (Selig \& Preacher, 2008) available at the website of the first author to compute a 95\% CI based on 20,000 simulated draws from the distributions for the $a_{1}$ and $b_{1}$ parameters. Figure 5 shows a simulated sampling distribution for the indirect effect $\left(a_{1} b_{1}\right)$. This histogram is automatically generated when computing MC CIs using the web-based calculator. The histogram shows a degree of positive skew consistent with the aforementioned nonnormal shape of distributions of products.

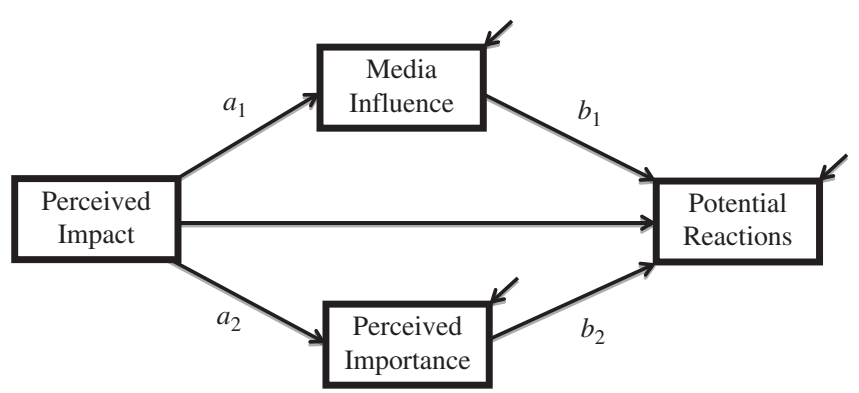

FIGURE 4 Diagram of the model from Tal-Or et al. (2010).

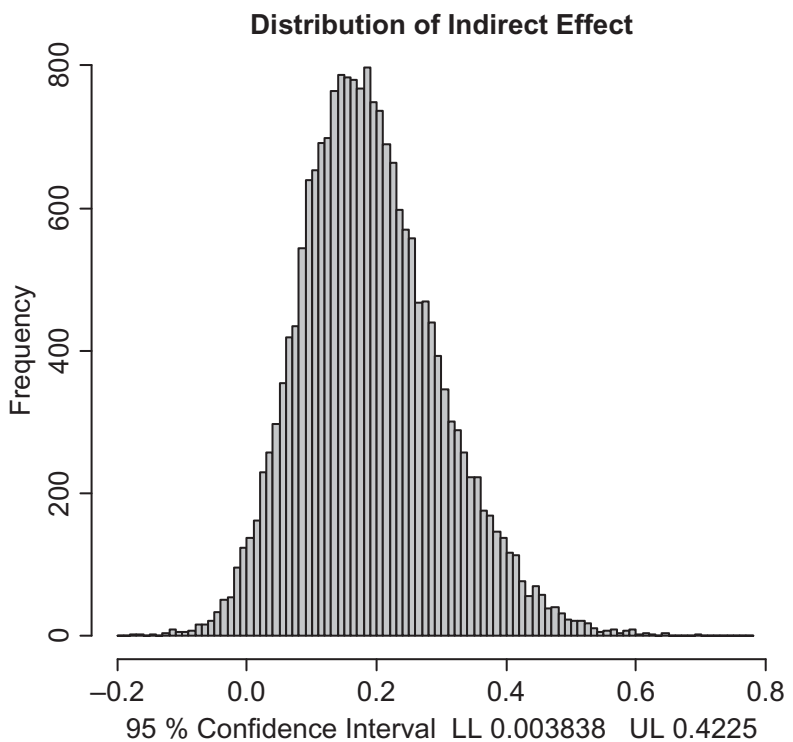

FIGURE 5 Simulated sampling distribution for the indirect effect $\left(a_{1} b_{1}\right)$. 
TABLE 2

95\% Confidence Intervals for the Indirect Effect of the Manipulation on Reactions as Mediated by Media Influence

\begin{tabular}{lrc}
\hline & $\begin{array}{r}95 \% \text { CIs for Indirect Effect } \\
\left(a_{1} b_{I}=0.189\right)\end{array}$ \\
\cline { 2 - 3 } Method & \multicolumn{1}{c}{$L L$} & $U L$ \\
\hline Monte Carlo & 0.004 & 0.423 \\
Delta Method & -0.015 & 0.393 \\
Percentile Bootstrap & 0.006 & 0.415 \\
Bias-Corrected Bootstrap & 0.016 & 0.436 \\
\hline
\end{tabular}

Next we used Mplus (Muthén \& Muthén, 1998-2010) to conduct a path analysis of the variables in Figure 4. For the path analysis we requested three different kinds of 95\% CIs that Mplus can produce. These three are based on: the delta method, the percentile bootstrap, and the bias corrected bootstrap. For this analysis we used 20,000 bootstrap resamples to compute the CIs. Table 2 shows the $95 \%$ CIs from all analyses. As can be seen from the 95\% CIs in Table 2, all intervals except for the symmetric CI based on the delta method exclude zero. Therefore, the authors' conclusion regarding the evidence for mediation would be similar when using the MC approach or either bootstrapping approach. The lower limit for the MC interval is lower than that for the two bootstrap CIs.

Next we used the same data to examine an indirect effect not considered by Tal-Or et al. (2010). This is the total indirect effect of perceived impact (X) on potential reactions (Y). The total indirect effect describes all of the influence perceived impact had on potential reactions as mediated by both perceived media influence $\left(\mathrm{M}_{1}\right)$ and perceived importance $\left(\mathrm{M}_{2}\right)$. The estimate of the total indirect effect can be calculated as follows: $\left(a_{1} b_{1}\right)+\left(a_{2} b_{2}\right)$. MacKinnon (2008) provides the following delta method formula for the standard error for such a total indirect effect:

$$
s_{\hat{a}_{1} \hat{b}_{1}+\hat{a}_{2} \hat{b}_{2}}=\sqrt{s_{\hat{a}_{1}}^{2} \hat{b}_{1}^{2}+s_{\hat{b}_{1}}^{2} \hat{a}_{1}^{2}+s_{\hat{a}_{2}}^{2} \hat{b}_{2}^{2}+s_{\hat{b}_{2}}^{2} \hat{a}_{2}^{2}+2 \hat{a}_{1} \hat{a}_{2} s_{\hat{b}_{1} \hat{b}_{2}}+2 \hat{b}_{1} \hat{b}_{2} s_{\hat{a}_{1} \hat{a}_{2}}}
$$

Here $s_{\hat{a}_{1} \hat{a}_{2}}$ and $s_{\hat{b}_{1} \hat{b}_{2}}$ describe the sample covariances between the two $a$ parameter estimates and the two $b$ parameter estimates, respectively.

Some SEM software packages such as Mplus allow the user to define new parameters that are functions of other model parameters. The software can then provide both estimates and standard errors for the new parameters. In this way, one could define the total indirect effect above as a new parameter and calculate a standard error and/or CI for the total indirect effect. In many situations it is also possible to use the MC approach to estimate CIs for the total indirect effect. This is done by simulating random draws from the distributions for the four parameters involved in the total indirect effect (i.e., $a_{1}, b_{1}, a_{2}, b_{2}$ ).

When using path analysis, there is no assumption that the parameter covariances are equal to zero, so it is possible that the four parameters that constitute the total indirect effect may have nonzero covariances. As can be seen in Equation 10, these covariances are needed to compute the standard error and are also important for the MC approach. In other words, it may be necessary 
to sample values of the coefficients from a multivariate normal distribution using the covariance matrix of the parameters as a population covariance matrix.

When it is necessary to know about the covariances of the parameter estimates constituting an indirect effect, there are two options available in addition to access to raw data. The first option requires the authors to report the asymptotic covariance matrix (ACM) of the parameter estimates, which can be obtained in most regression and SEM packages. Given current reporting norms, we believe this will be a rare occurrence. The second option requires the authors to report the covariance matrix for the variables used in the analysis. It is then possible to use the covariance matrix in an SEM software package to replicate the analyses in the report. Many software packages will then give the user the option of outputting the ACM. This covariance matrix can then be used to simulate random values from a multivariate normal distribution of the parameters.

To demonstrate the use of MC CIs for the total indirect effect, we started with the correlations and standard deviations for the variables used in the model. The correlations were not reported in the Tal-Or et al. (2010) article, but many times this information is made available in a published report. We then used only these summary descriptive statistics to conduct a path analysis and output the parameter covariance matrix. The largest covariance was that between parameters $b_{1}$ and $b_{2}$. However, this covariance was exceptionally small (0.39E-17). Given the negligible covariances among the parameter estimates, we chose to compute the MC CI as if the four parameters were orthogonal. This both simplified the simulation and made the estimation of the MC CI more closely resemble the scenario in which authors report only parameter estimates and standard errors. We constructed the MC CI for the total indirect effect by simulating random draws from the four normal distributions for each of the constituent parameters of the total indirect effect (i.e., $a_{1}, b_{1}, a_{2}, b_{2}$ ). These parameters and the associated standard errors are as follows: $a_{1}=0.48, S E_{a 1}=0.24, b_{1}=0.40, S E_{b 1}=0.09, a_{2}=0.62, S E_{a_{2}}=0.31, b_{2}=0.32, S E_{b_{2}}=0.07$. We used $\mathrm{R}$ to simulate the random draws, compute the total indirect effect for each repetition, and compute the $95 \%$ CI based on the distribution of 20,000 simulated total indirect effects. The $\mathrm{R}$ code for this analysis is included in the Appendix. As before, we used the results from a path analysis of the original data to compute the other CIs. The four $95 \%$ CIs for the total indirect effect are shown in Table 3. These four CIs all support the indirect effect of the manipulation on reactions.

TABLE 3

95\% Confidence Intervals for the Total Indirect Effect of the Manipulation on Reactions as Mediated by Both Media Influence and Perceived Importance

\begin{tabular}{lcc}
\hline & $\begin{array}{r}95 \% \text { CIs for Total Indirect Effect } \\
\left(a_{1} b_{1}+a_{2} b_{2}=0.392\right)\end{array}$ \\
\cline { 2 - 3 } Method & $L L$ & $U L$ \\
\hline Monte Carlo & 0.109 & 0.716 \\
Delta Method & 0.067 & 0.718 \\
Percentile Bootstrap & 0.086 & 0.741 \\
Bias-Corrected & 0.087 & 0.743 \\
$\quad$ Bootstrap & & \\
\hline
\end{tabular}




\section{DISCUSSION}

In this article we focused on CI construction, with an emphasis on evaluating the utility of the Monte Carlo method. The relative success of different CI construction methods can be assessed in terms of coverage with respect to the nominal confidence level, symmetry (i.e., the population parameter should be excluded equally on either side), and width (shorter intervals are preferred). The MC method performed on par with bootstrap and distribution of the product methods that have been found to perform well in simulation studies. We applied the MC method of constructing CIs in two examples drawn from a study by Tal-Or et al. (2010)—one involving a one-mediator indirect effect and a second involving a total indirect effect from a model with two mediatorsalong with delta method and bootstrap CIs for comparison. The MC CIs were comparable to bootstrap CIs and led to the same conclusions.

The advantages associated with the MC approach over other asymmetric CI methods include enhanced precision due to smoothness of the sampling distribution and its usefulness when only summary data are available. Relative to other methods that require repeated model-fitting, the MC method is very fast. But when should the MC method be preferred to well-performing competing methods such as bootstrap and DP methods? We believe that if nonparametric bootstrap or DP methods are feasible options, then researchers should use them. However, there are many situations in which these methods will not be feasible, in which case the MC method should be considered a viable and competitive method for constructing CIs for simple and complex indirect effects. For example, there is no agreed-upon best way to employ bootstrapping in multilevel modeling, although RB bootstrap seems to be a good option (van der Leeden et al., 2008; Wang et al., 2006). Even so, multilevel modeling software currently does not make it easy to estimate even simple indirect effects. Until one bootstrap method emerges as best in the multilevel context, MC may be the only viable method (see Bauer, Preacher, \& Gil, 2006, and Preacher, Zyphur, \& Zhang, 2010 for uses of this method in the multilevel context). Models that involve large samples and/or intensive numerical integration to obtain parameter estimates (e.g., mixture models with multiple binary outcomes) often make nonparametric and parametric bootstrapping-both of which involve fitting the model $B$ times-too time-consuming to be feasible in practice. In these situations, $\mathrm{MC}$ is a practical alternative because no further model-fitting is required. The DP method currently has been implemented only for simple indirect effects consisting of products of two coefficients, which may or may not covary. However, in many modeling contexts more complex indirect effects are of interest. Examples include total indirect effects in multiple mediator models (MacKinnon, 2000; Preacher \& Hayes, 2008) and virtually any indirect effect in panel mediation models (Cole \& Maxwell, 2003). In these situations the DP method cannot currently be used, but the MC method can.

Several software options are available to researchers who wish to use the MC method of constructing CIs for simple and complex indirect effects. The MC method was used by Bauer et al. (2006) when assessing mediation in multilevel modeling using SAS, and by Preacher et al. (2010) in the context of assessing mediation with multilevel SEM in Mplus. The authors have made syntax for both applications available online. ${ }^{2}$ It is implemented in online R calculators for single-level and (some) multilevel models for mediation (Preacher \& Selig, 2010; Selig \& Preacher, 2008), and in the MEDIATE macro for SPSS and (soon) SAS by Hayes and

\footnotetext{
${ }^{2}$ See http://quantpsy.org/
} 
Preacher (under review) for use in mediation models with continuous, binary, or multicategorical predictors. ${ }^{3}$ The MC method is employed in the context of causal mediation analysis using the R package mediation (Imai, Keele, Tingley, \& Yamamoto, 2010; see also Imai, Keele, \& Tingley, 2010, pp. 316-317) and in a similar package for Stata (Hicks \& Tingley, 2011). The method is easy to implement in Microsoft Excel for simple mediation models, and use of Excel is feasible in larger models.

One limitation of the present study is that we considered only one confidence level (95\%). Future studies should consider the performance of various CI construction methods with different confidence levels, especially in light of Efron's (1988) warning that bootstrap CIs may not perform so well at more extreme coverage probabilities. Future studies also should use more than 1,000 bootstrap resamples to determine empirical Type I error rates and power with greater precision, particularly for the bias-corrected bootstrap methods (Williams, 2004). Our findings are also circumscribed by our particular choices of population parameters $a, b$, and $c$ and sample sizes $N$. Future research should consider other values. We also considered only the simple mediation model because of its simplicity. Future research should examine the performance of the various $\mathrm{CI}$ construction methods in more complex modeling contexts, where differences in performance across CI methods may be even more pronounced. Finally, in this article we did not consider Bayesian credible intervals for indirect effects, another promising method for obtaining asymmetric intervals for indirect effects (Biesanz et al., 2010; Yuan \& MacKinnon, 2009).

We did not address significance testing in this article. CIs often are used to test null hypotheses in mediation analysis and other settings, but it is worth noting that the most widely accepted methods of constructing CIs for indirect effects are not necessarily the best methods for testing null hypotheses. In fact, there are logical problems with using CIs based on sampling distributions for testing null hypotheses, which are more properly tested using null distributions rather than sampling distributions (Biesanz et al., 2010). Tests based on null distributions and CIs from sampling distributions will not always agree on what conclusion should be drawn about the null hypothesis, so caution is warranted in using CIs for this purpose. Future research may address the use of MC methods to create null distributions, which are more suitable for testing point null hypotheses in mediation analysis.

\section{ACKNOWLEDGEMENTS}

The authors wish to thank Nurit Tal-Or, Jonathan Cohen, Yariv Tsfati, and Albert C. Gunther for use of their data in illustrative examples. We also thank Andrew Hayes for helpful comments.

\section{REFERENCES}

Aroian, L. A. (1947). The probability function of the product of two normally distributed variables. Annals of Mathematical Statistics, 18, 265-271.

Baron, R. M., \& Kenny, D. A. (1986). The moderator-mediator variable distinction in social psychological research: Conceptual, strategic, and statistical considerations. Journal of Personality and Social Psychology, 51, 1173-1182.

\footnotetext{
${ }^{3}$ See http://afhayes.com/
} 
Bauer, D. J., Preacher, K. J., \& Gil, K. M. (2006). Conceptualizing and testing random indirect effects and moderated mediation in multilevel models: New procedures and recommendations. Psychological Methods, 11, 142-163.

Biesanz, J. C., Falk, C., \& Savalei, V. (2010). Assessing mediational models: Testing and interval estimation for indirect effects. Multivariate Behavioral Research, 45, 661-701. doi: 10.1080/00273171.2010.498292

Bollen, K. A., \& Stine, R. (1990). Direct and indirect effects: Classical and bootstrap estimates of variability. Sociological Methodology, 20, 115-140.

Chernick, M. R. (2008). Bootstrap methods: A guide for practitioners and researchers (2nd ed.). Hoboken, NJ: Wiley.

Cheung, M. W. L. (2007). Comparison of approaches to constructing confidence intervals for mediating effects using structural equation models. Structural Equation Modeling, 14, 227-246.

Cole, D. A., \& Maxwell, S. E. (2003). Testing mediational models with longitudinal data: Questions and tips in the use of structural equation modeling. Journal of Abnormal Psychology, 112, 558-577.

Craig, C. C. (1936). On the frequency function of xy. Annals of Mathematical Statistics, 7, 1-15.

Davison, A. C., \& Hinkley, D. V. (1997). Bootstrap methods and their application. Cambridge, UK: Cambridge University Press.

DiCiccio, T. J., \& Efron, B. (1996). Bootstrap confidence intervals (with discussion). Statistical Science, 11, 189-228.

DiCiccio, T. J., \& Romano, J. P. (1989). The automatic percentile method: Accurate confidence limits in parametric models. Canadian Journal of Statistics, 17, 155-169.

Efron, B. (1979). Bootstrap methods. Annals of Statistics, 7, 1-26.

Efron, B. (1980). The jackknife, the bootstrap, and other resampling plans (Technical Report No. 63). Stanford, CA: Department of Statistics, Stanford University.

Efron, B. (1981). Nonparametric estimates of standard error: the jackknife, the bootstrap, and other resampling methods. Biometrika, 68, 589-599.

Efron, B. (1982). The jackknife, the bootstrap and other resampling plans. Philadelphia, PA: Society of Industrial and Applied Mathematics CBMS-NSF Monographs, 38.

Efron, B. (1987). Better bootstrap confidence intervals (with discussion). Journal of the American Statistical Association, $82,171-185$.

Efron, B. (1988). Bootstrap confidence intervals: Good or bad? Psychological Bulletin, 104, 293-296.

Efron, B., \& Tibshirani, R. (1986). Bootstrap methods for standard errors, confidence intervals, and other measures of statistical accuracy. Statistical Science, 1, 54-75.

Efron, B., \& Tibshirani, R. J. (1993). An introduction to the bootstrap. New York, NY: Chapman \& Hall.

Freedman, D. (1981). Bootstrapping regression models. Annals of Statistics, 9, 1218-1228.

Fritz, M. S., \& MacKinnon, D. P. (2007). Required sample size to detect the mediated effect. Psychological Science, 18, 233-239.

Fritz, M. S., Taylor, A. B., \& MacKinnon, D. P. (2012). Explanation of two anomalous results in statistical mediation analysis. Multivariate Behavioral Research, 47, 61-87.

Hall, P. (1988). Theoretical comparison of bootstrap confidence intervals. Annals of Statistics, 16, 927-985.

Hayes, A. F., \& Preacher, K. J. (2011). Indirect and direct effects of a multicategorical causal agent in statistical mediation analysis, (under review).

Hicks, R., \& Tingley, D. (2011). Causal mediation analysis. Stata Journal, 11, 609-615.

Imai, K., Keele, L., \& Tingley, D. (2010). A general approach to causal mediation analysis. Psychological Methods, 15, 309-334.

Imai, K., Keele, L., Tingley, D., \& Yamamoto, T. (2010). Causal mediation analysis using R. In H. D. Vinod (Ed.), Advances in social science research using $R$ (pp. 129-154). New York, NY: Springer.

Kaiser, H. F., \& Dickman, K. (1962). Sample and population score matrices and sample correlation matrices from an arbitrary population correlation matrix. Psychometrika, 27, 179-182.

Lockwood, C. M., \& MacKinnon, D. P. (1998). Bootstrapping the standard error of the mediated effect. In Proceedings of the 23rd Annual Meeting of SAS Users Group International (pp. 997-1002). Cary, NC: SAS Institute, Inc.

MacKinnon, D. P. (2000). Contrasts in multiple mediator models. In J. Rose, L. Chassin, C. C. Presson, \& S. J. Sherman (Eds.), Multivariate applications in substance use research: New methods for new questions (pp. 141-160). Mahwah, NJ: Erlbaum.

MacKinnon, D. P. (2008). Introduction to statistical mediation analysis. New York, NY: Erlbaum.

MacKinnon, D. P., Fritz, M. S., Williams, J., \& Lockwood, C. M. (2007). Distribution of the product confidence limits for the indirect effect: Program PRODCLIN. Behavior Research Methods, 39, 384-389. 
MacKinnon, D. P., Lockwood, C. M., Hoffman, J. M., West, S. G., \& Sheets, V. (2002). A comparison of methods to test mediation and other intervening variable effects. Psychological Methods, 7, 83-104.

MacKinnon, D. P., Lockwood, C. M., \& Williams, J. (2004). Confidence limits for the indirect effect: Distribution of the product and resampling methods. Multivariate Behavioral Research, 39, 99-128.

Meeker, W. Q., Cornwell, L. W., \& Aroian, L. A. (1981). Selected tables in mathematical statistics: The product of two normally distributed random variables. Providence, RI: American Mathematical Society.

Muthén, B. O. (2011). Applications of causally defined direct and indirect effects in mediation analysis using SEM in Mplus. Unpublished manuscript.

Muthén, L. K., \& Muthén, B. O. (1998-2010). Mplus user's guide (6th ed.). Los Angeles, CA: Muthén \& Muthén.

Pearl, J. (2010). The foundations of causal inference. Sociological Methodology, 40, 75-149.

Pituch, K. A., \& Stapleton, L. M. (2008). The performance of methods to test upper-level mediation in the presence of nonnormal data. Multivariate Behavioral Research, 43, 237-267.

Pituch, K. A., Stapleton, L. M., \& Kang, J. Y. (2006). A comparison of single sample and bootstrap methods to assess mediation in cluster randomized trials. Multivariate Behavioral Research, 41, 367-400.

Pituch, K. A., Whittaker, T. A., \& Stapleton, L. M. (2005). A comparison of methods to test for mediation in multisite experiments. Multivariate Behavioral Research, 40, 1-23.

Polansky, A. M. (1999). Upper bounds on the true coverage probability of bootstrap percentile type confidence intervals. The American Statistician, 53, 362-369.

Preacher, K. J., \& Hayes, A. F. (2004). SPSS and SAS procedures for estimating indirect effects in simple mediation models. Behavior Research Methods, Instruments, \& Computers, 36, 717-731.

Preacher, K. J., \& Hayes, A. F. (2008). Asymptotic and resampling strategies for assessing and comparing indirect effects in multiple mediator models. Behavior Research Methods, 40, 879-891.

Preacher, K. J., Rucker, D. D., \& Hayes, A. F. (2007). Assessing moderated mediation hypotheses: Theory, methods, and prescriptions. Multivariate Behavioral Research, 42, 185-227.

Preacher, K. J., \& Selig, J. P. (2010, July). Monte Carlo method for assessing multilevel mediation: An interactive tool for creating confidence intervals for indirect effects in 1-1-1 multilevel models [Computer software]. Available from http://quantpsy.org/

Preacher, K. J., Zyphur, M. J., \& Zhang, Z. (2010). A general multilevel SEM framework for assessing multilevel mediation. Psychological Methods, 15, 209-233.

Schenker, N. (1985). Qualms about bootstrap confidence intervals. Journal of the American Statistical Association, 80 , $360-361$

Selig, J. P., \& Preacher, K. J. (2008, June). Monte Carlo method for assessing mediation: An interactive tool for creating confidence intervals for indirect effects [Computer software]. Available from http://quantpsy.org/

Shrout, P. E., \& Bolger, N. (2002). Mediation in experimental and nonexperimental studies: New procedures and recommendations. Psychological Methods, 7, 422-445.

Sobel, M. E. (1982). Asymptotic intervals for indirect effects in structural equations models. In S. Leinhart (Ed.), Sociological methodology 1982 (pp. 290-312.) San Francisco, CA: Jossey-Bass.

Stine, R. A. (1989). An introduction to bootstrap methods: Examples and ideas. Sociological Methods in Research, 16 , 243-291.

Tal-Or, N., Cohen, J., Tsfati, Y., \& Gunther, A. C. (2010). Testing causal direction in the influence of presumed media influence. Communication Research, 37, 801-824.

van der Leeden, R., Meijer, E., \& Busing, F. M. T. A. (2008). Resampling multilevel models. In J. de Leeuw \& E. Meijer (Eds.), Handbook of multilevel analysis (pp. 401-433). New York, NY: Springer.

Wang, J., Carpenter, J. R., \& Kepler, M. A. (2006). Using SAS to conduct nonparametric residual bootstrap multilevel modeling with a small number of groups. Computer Methods and Programs in Biomedicine, 82, 130-143.

Williams, J. (2004). Resampling and distribution of the product methods for testing indirect effects in complex models. (Unpublished doctoral dissertation). Tempe, Arizona: Arizona State University.

Williams, J., \& MacKinnon, D. P. (2008). Resampling and distribution of the product methods for testing indirect effects in complex models. Structural Equation Modeling, 15, 23-51. doi: 10.1080/10705510701758166

Yuan, Y., \& MacKinnon, D. P. (2009). Bayesian mediation analysis. Psychological Methods, 14, 301-322.

Zhang, Z., \& Wang, L. (2008). Methods for evaluating mediation effects: Rationale and comparison. In K. Shigemasu, A. Okada, T. Imaizumi, \& T. Hoshino (Eds.), New trends in psychometrics (pp. 595-604). Tokyo, Japan: Universal Academy Press. 


\section{APPENDIX A: R CODE FOR TOTAL INDIRECT EFFECT EXAMPLE}

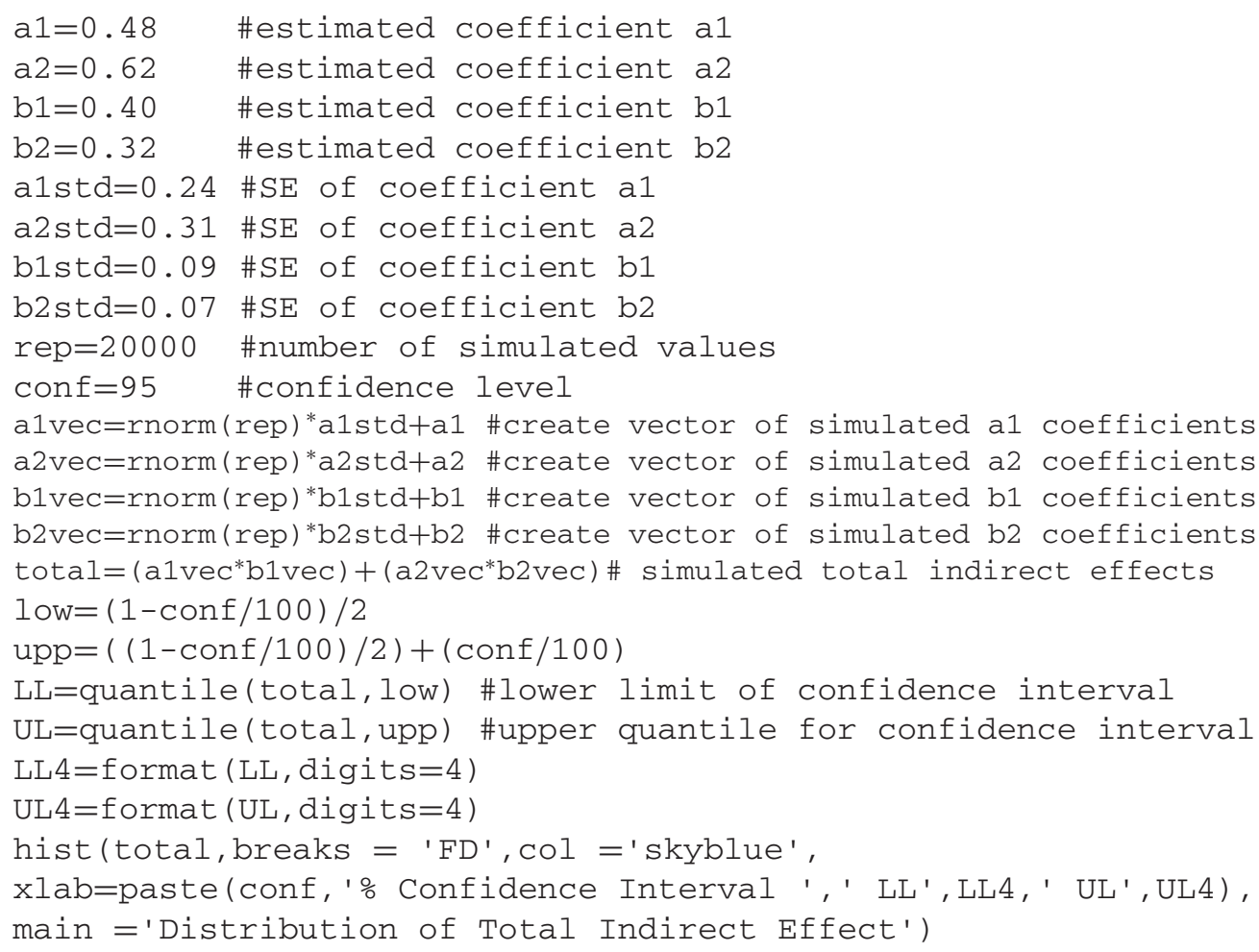

